\title{
DESIGN OF CODE ACQUISITION METHOD OF FHSS SYSTEM
}

\author{
MOHANAD ABDULHAMID ${ }^{1}$, GABRIEL OGWALI ${ }^{2}$ \\ ${ }^{1}$ AL-Hikma University, Iraq, ${ }^{2}$ University of Nairobi, Kenya \\ E-mail: moh1hamid@yahoo.com, researcher12018@yahoo.com
}

\begin{abstract}
Frequency hopping spread spectrum (FHSS) communication systems offer significant performance advantages in view of their low probability of intercept, improved performance in multipath fading environments and their ability to avoid interference by hopping into low interference frequency channels. For the transmitted sequence to be correctly received and demodulated, the frequency hop sequence used at the receiver should be similar to that employed in the transmitter. Code acquisition in frequency hopping attempts to address this problem by providing a frequency hop pattern at the receiver that is nearly identical to that used at the transmitter. Code acquisition brings the alignment between the transmitter and the receiver hop pattern to at least one hop period. The main objective of this paper is to design and demonstrate a FHSS code acquisition method. The type of channel used is Additive White Gaussian Noise (AWGN).
\end{abstract}

Keywords: FHSS; code acquisition method; AWGN

\section{INTRODUCTION}

The main problem in telecommunications is generating a message and ensuring it reaches the recipient in the desired form. While this may sound like a rather mundane task, numerous constraints imposed by factors such as channel imperfections offer a tremendous challenge towards achieving this goal. Nevertheless, a number of techniques such as information coding seem to address this challenge [1].

This is not the only challenge that may affect the sending of messages over a communication channel. One other challenge is how to efficiently utilize the available channel bandwidth. One such technique that has been successfully utilized in improving channel efficiency toward this front is frequency division multiplexing [2].

The period during which the Second World War took place witnessed numerous challenges as well as improvements in the way telecommunications takes place. The primary interest at the time was not merely getting the message to the recipient. Military espionage was a real threat and thus information integrity had to be guaranteed.

Information integrity was guaranteed in two main ways. The first step was ensuring that a potential jammer does not even get to know that information is even being transmitted in the first place. The second step was to transmit information in such a way as to ensure that only the sender knows where to get the information from. All this is effected by means of some code or algorithm [2, 3].

Spread spectrum communication schemes ensure that the two mentioned criteria are met [4,5]. Simply put, a spread spectrum communication scheme is a means of communication under which a message is transmitted at a bandwidth that is several times the size of the original message bandwidth. There are two major classes of spread spectrum systems, these are [5]:

1 - Pure spread spectrum systems

2 - Hybrid spread spectrum systems

Under pure spread spectrum systems the following three spread spectrum systems are obtained:

1 - Direct Sequence Spread Spectrum (DSSS);

2 - Frequency Hopping Spread Spectrum (FHSS);

3 - Time Hopping Spread Spectrum (THSS).

Hybrid systems are obtained by combining two or more of the pure spread spectrum systems. The primary goal of hybrid systems is to tap into and exploit the unique advantages of each of the pure spread spectrum systems used. While doing this, hybrid systems combat each of the individual shortcomings of each of the spread spectrum systems $[5,6]$.

As a case in point, consider a hybrid Direct SequenceFrequency Hopping Spread Spectrum (DS/FH) system. When employed in a channel with interference in specific bands, a frequency hopping algorithm can be employed to effect hops only into suitable bands. After hopping into the desirable bands, a direct sequence spread can be effected. This improves the spreading gain of the hybrid system and as a result improves the system performance in channels where most of the noise occurs in few frequency bands which can be predicted. The main hybrid spread spectrum systems are as follows:

1 - DS/FH

2 - DS/TH

3 - FH/TH

4 - DS/FH/TH

Spread spectrum systems use pseudo random signals to effect the spreading. These spreading sequences are referred to as pseudo noise (PN) sequences or PN codes. $\mathrm{PN}$ sequences is generated using linear feedback shift registers (LFSR). PN sequences have a number of properties that make them reliable as compared to other codes during acquisition $[7,8]$. 
In FHSS, code synchronization involves two steps namely; code acquisition and code tracking. The code acquisition phase involves bringing the $\mathrm{PN}$ sequence to at least within a time period of the PN sequence used to generate the carrier frequency at the receiver. Code tracking involves further refining the acquired $\mathrm{PN}$ sequence improving its accuracy to less than half the time period of the transmitted signal $[9,10]$.

There are different methods of code acquisition but in this paper, serial search code acquisition is considered. In a serial search acquisition method, the received spreading PN sequence used at the transmitter is correlated with the one that is used at the receiver and periodically delayed until a set threshold is attained. Once this threshold is attained, acquisition is declared and the declaration of acquisition initiates tracking [11].

\section{DESIGN METHODOLOGY}

Code acquisition in a FHSS is simulated using the MATLAB Simulink simulation software. Appropriate Simulink block sets are used and their parameters varied to conform to design specifications. For generation of the hopping codes, PN sequences are used due to their correlation properties that allow for easier acquisition. For simulation purposes the AWGN channel is simulated. The Simulink block diagram in Figure 1 outlines the complete design.
A few of the various design aspects of the common block parameters are designed in the next few paragraphs. The rationale for arriving at each of the design parameters is also discussed.

\subsection{Bernoulli Binary Generator}

The Bernoulli binary generator is chosen as the simulation data source since it outputs a random sequence of 0 and 1 bit sequences. The probability of getting a 0 and a 1 are all set to be equal i.e. both have a probability of 0.5 .

The bit rate for the Bernoulli binary generator is set at 10,000 bits per second. Before the generated Bernoulli sequence is mixed with the carrier frequency generated through the 8-Frequency-Shift-Keying (FSK) modulator, it is first 2-FSK modulated.

At the receiver, a 2-FSK demodulator is used for demodulation. If the demodulation process is effective, an exact replica of what has been transmitted should be received.

\subsection{PN Sequence}

A 4-stage LFSR with a PN sequence length of 15 is used. The LFSR used is a maximal sequence LFSR. Similar PN sequence generators are used at both the transmitter and the receiver. The output bit rate for both

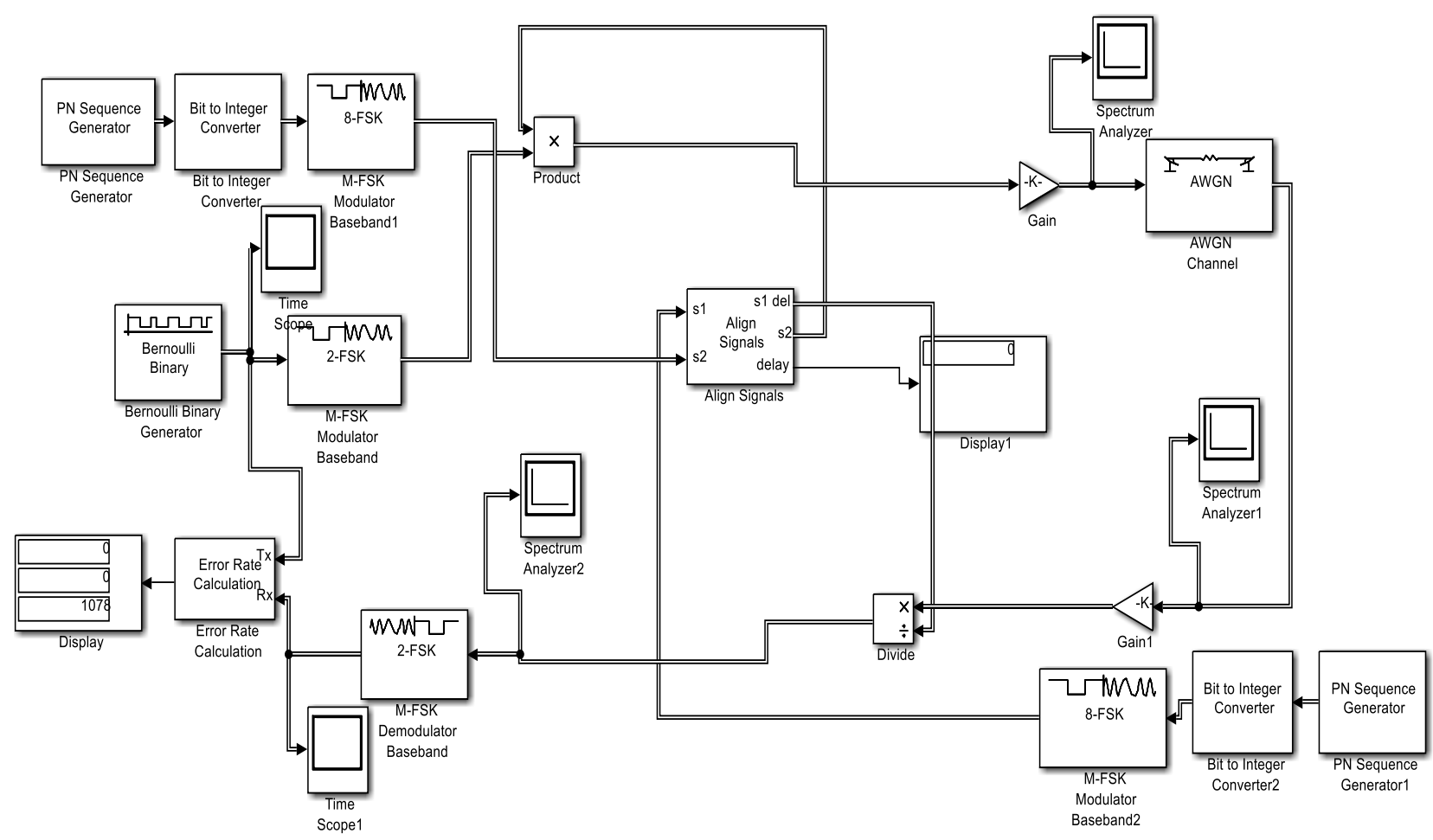

PN semence generators is set at 90,000 bits per second.

Figure 1. Simulation block for serial search acquisition for a FHSS system 
The generated bits from the PN sequence generator are then clustered into groups of 3 bits and a bit to integer converter is used to generate eight possible integer values ranging from 0 to 7 . The output of the bit to integer converter is then fed to an 8-FSK modulator which then outputs the different carrier frequencies that are used to effect frequency hopping.

Since the output bit rate from the PN sequence generator is 90,000 bits per second and they are grouped into clusters of 3 , there are 30,000 clusters generated per second. Therefore, ideally, the output frequency of the 8FSK modulator varies 3 times per symbol of the data sequence generated by the Bernoulli Binary Generator.

\subsection{8-FSK Modulator}

The 8-FSK modulator is set to have a carrier frequency separation of $100 \mathrm{kHz}$. The eight possible carrier frequencies therefore range from $100 \mathrm{kHz}$ to $800 \mathrm{kHz}$.

\subsection{Align Signals Block(Serial Search Acquisition)}

The align signals block is the block that performs the serial search acquisition. The align signals block compares two signals by correlating them and then adjusts the signal to be adjusted based on the correlation peak. PN sequences offer a lot of demodulation ease since they have specific correlation peaks.

In the design highlighted above, before the PN sequence that is used at the transmitter is transmitted to the align signal block at the receiver, it is first 8-FSK modulated. The PN sequence at the receiver is also 8-FSK modulated and after this the PN sequence at the receiver is aligned to that received from the transmitter. The align signal block periodically delays the PN sequence at the receiver until it is aligned to the one at the receiver.

\subsection{AWGN Channel}

In this paper, AWGN channel is considered as a transmission channel. This type of channel adds additive white Gaussian noise only to the transmitted signal.

\section{SIMULATION RESULTS}

\subsection{PN Sequence Results}

The properties of the PN sequence used are first tested for their randomness properties. Figure 2 shows the timing diagram for a 4-stage LFSR maximal length sequence.

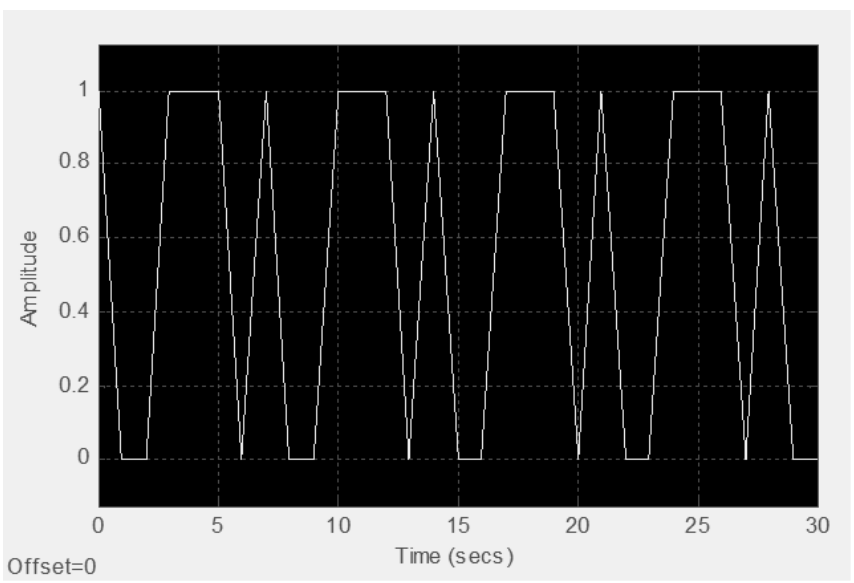

Figure 2. PN sequence timing diagram

The sample time for the PN sequence is set at $1 \mathrm{sec}$ just for illustrative purposes. For a larger sampling frequency, the basic properties will remain the same. Since the sample time is $1 \mathrm{sec}$, and a maximal length sequence is utilized, the expected PN sequence period is $15 \mathrm{sec}$. From the graph, after a time interval of roughly $15 \mathrm{sec}$ the sequence repeats itself.

The correlation properties of the PN sequences are also tested. From Figure 2 above, if a sequence similar to the one in the Figure 2 is compared for the number of agreements and disagreements for every $1 \mathrm{sec}$ interval for $15 \mathrm{sec}$, the number of agreements would be 15 while the number of disagreements would be zero. This would yield the maximum correlation value. Any shift by a value that is not a multiple of $15 \mathrm{sec}$ would increase the number of disagreements between the un-delayed and delayed version of the PN sequence. This would effectively reduce the correlation between the two signals.

Figure 3 shows the correlation output for two perfectly aligned PN sequences while Figure4 shows the correlation output between two PN sequences with a misalignment of $1 \mathrm{sec}$ between each other.

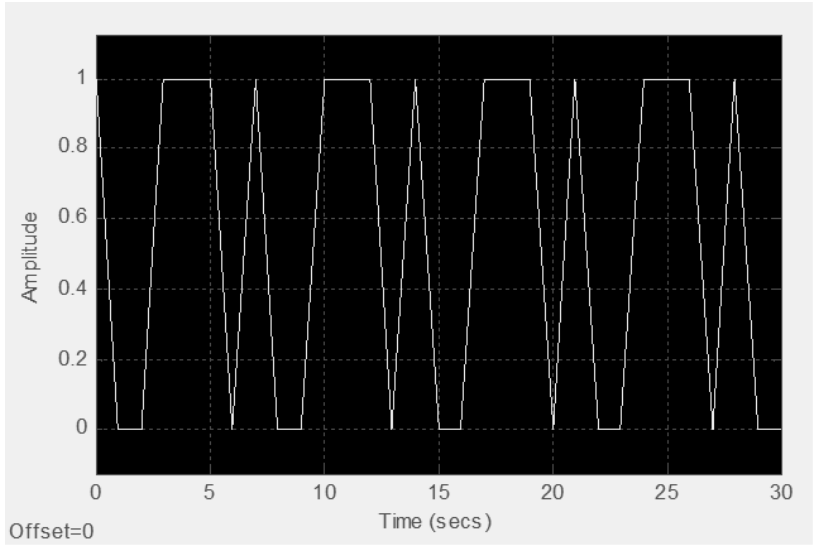

Figure 3. Correlation between two perfectly aligned PN sequences 


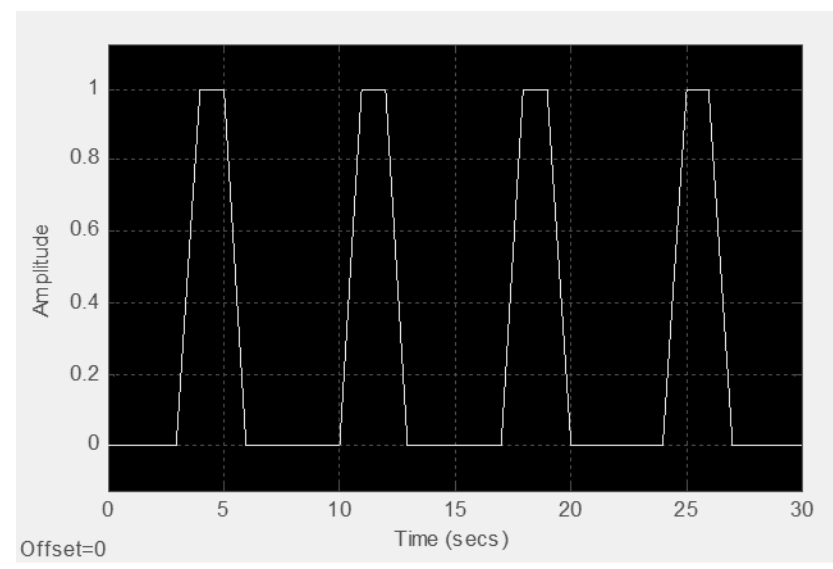

Figure 4. Correlation between two PN sequences with a misalignment of 1 sec

Accumulating the output of Figure 3 over a period of $15 \mathrm{sec}$ and doing the same thing for the output in Figure 4 , the output of Figure 3 yields a larger accumulated value than that of Figure 4 . The output of Figure 4 represents the situation for all other cyclic shifts. It is therefore evident that the PN sequence has a finite accumulated correlation maximum only when two PN sequences generated by the same generator polynomial are in synchronization.

\subsection{Results for the Transmission Over AWGN Channel}

The continuous time transmitted and received Bernoulli binary sequence is compared. Their time scopes appear as shown in Figure 5 and Figure 6 below. In this case the bit error rate is found to be 0 . In case a unit time delay is effected in the loop, the two spreading signals are aligned and the bit error rate is in the range of $10^{-5}$.

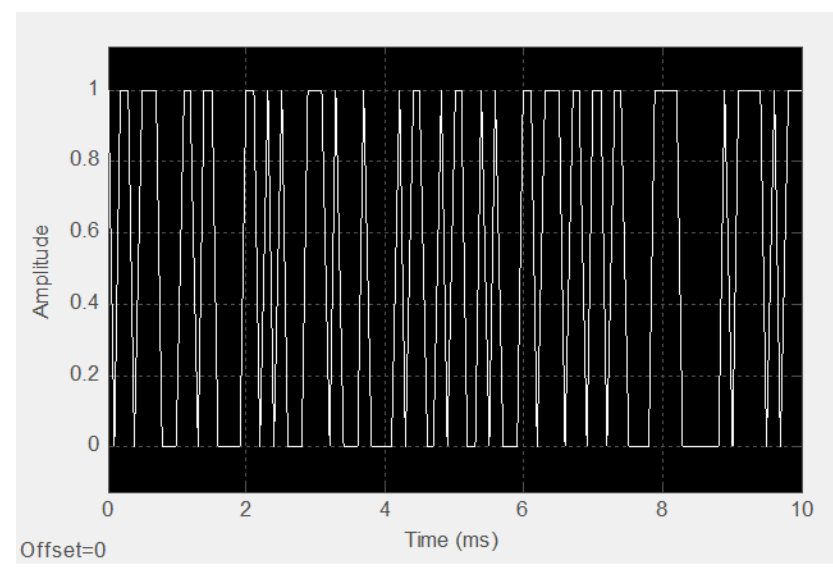

Figure 5. Transmitted Bernoulli binary signal

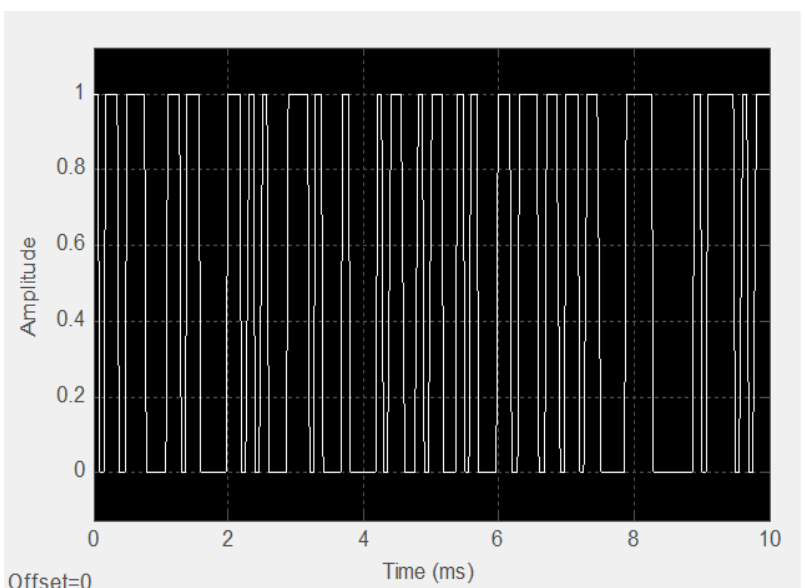

Figure 6. Received Bernoulli binary signal

Figure 7, Figure 8, and Figure 9 show the frequency spectrum of the transmitted signal, the frequency spectrum after frequency hopping and the frequency spectrum after code acquisition respectively.

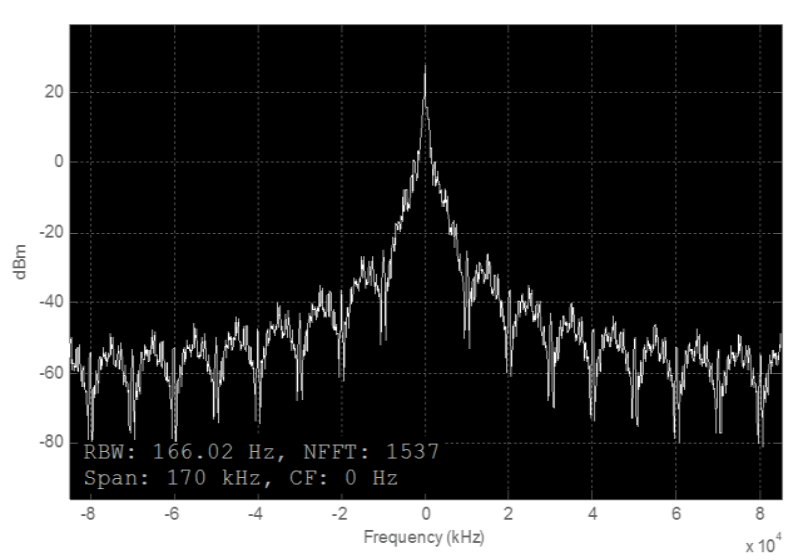

Figure 7. Frequency spectrum of the transmitted signal after 2-FSK modulation

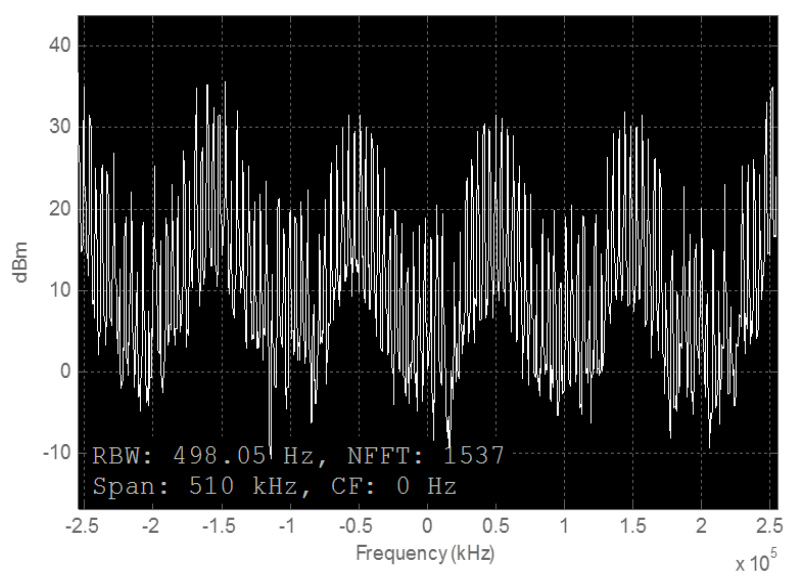

Figure 8. Frequency spectrum after frequency hopping 


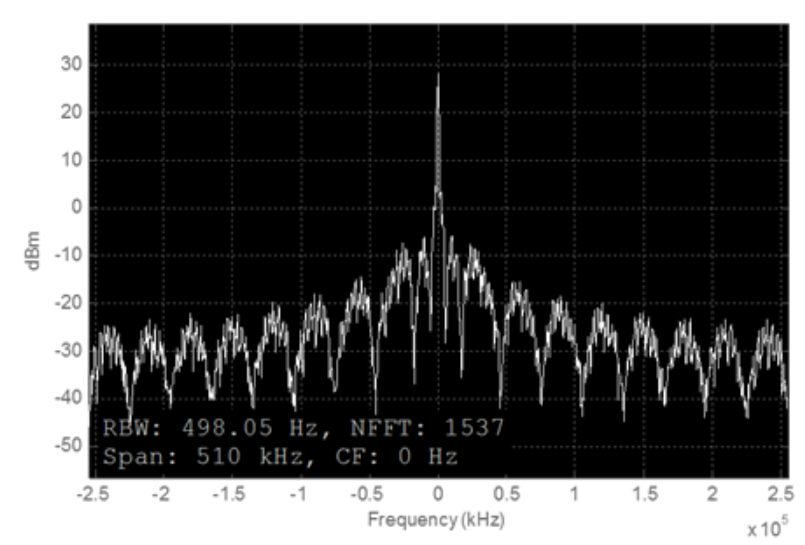

Figure 9. Frequency spectrum after code acquisition

\section{CONCLUSIONS AND DISCUSSION}

In this paper, spread spectrum communication systems were studied and the importance of code acquisition in frequency hopping spread spectrum communication systems was observed. The need for code acquisition in FHSS systems arises due to clocking instabilities at the receiver and uncertainty in the propagation delay of signals. Code acquisition is a precursor to the fine alignment process of code tracking. Therefore, the results obtained from code acquisition process are not perfect but fine alignment improves the accuracy. A serial search acquisition system was designed and simulated with the performance under a range of channel conditions getting noted. The reason for settling for a serial search acquisition system is informed by its use of fewer components as compared to the matched filter acquisition technique which requires a bank of bandpass filters.

\section{REFERENCES}

[1] B. Sklar, Digital communications: fundamentals and applications, Second Edition, Upper Saddle River, New Jersey: Prentice Hall PTR, 2009.

[2] W. Stallings, Data and computer communications, Eighth Edition, Upper Saddle River, New Jersey: Pearson Education Inc., 2007.

[3] M. Simon, J. Omura, R. Scholtz and B. Levitt, Spread spectrum communications handbook, Electronic Edition, McGraw-Hill Inc., 2004.

[4] D. Torrieri, Principles of spread spectrum communication systems, Springer Science Business Media Inc., 2005.

[5] A. Viterbi, CDMA: principles of spread spectrum communications, Addison-Wesley Publishing Company, 1995.

[6] H. Zepernick and A. Finger, Pseudo random signal processing, theory and application, John Wiley \& Sons, 2005.

[7] Z. Bo, S. Ding, and T. Elhabian, Study on fast acquisition of hybrid DS/ FHSS, Chinese Journal of Aeronautics, Vol.18, No.2, PP.161-165, 2005.

[8] G. Ogwali, Acquisition of frequency hopping spread spectrum signals, B.Sc. Graduation Project, University of Nairobi, Kenya, 2009.
[9] C. Chung and P. Huang, Effects of fading and partial-band noise jamming on a fast FH/BFSK acquisition receiver with noise-normalization combination, IEEE Transactions on Communications, Vol. 44, No.1, PP. 94-104, 1996.

[10] A. Elezabi and E. Sourour, Frequency hopping spread-spectrum acquisition under partial band interference and fading channels, IEEE Vehicular Technology Conference, USA, 2005.

[11] X. Zhao, H. Quan, and P. Cui, Research on synchronization technology of frequency hopping communication system, 6th International Conference on Computer-Aided Design, Manufacturing, Modeling and Simulation (CDMMS), 2018. 